\title{
Quantification of Global Tortuosity in Retinal Blood Vessels
}

\author{
Astri Handayani", Tesi Dwi Nafia ${ }^{\#}$, and Tati Rajab Mengko \\ ${ }^{\#}$ School of Electrical Engineering and Informatics, Bandung Institute of Technology, Jl. Ganesha No. 10, Bandung, 40132, Indonesia \\ E-mail: a.handayani@ieee.org,tesidwi@gmail.com,tatimengko@itb.ac.id
}

\begin{abstract}
Tortuosity is a parameter that indicates the tendency of a blood vessel segment to contain multiple twists and turns. Chronic hemodynamic changes in the body due to diabetes and hypertension will manifest as increased retinal vascular tortuosity, rendering tortuosity as a suitable indicator for diabetic and hypertensive retinopathy. Retinal tortuosity may be evaluated locally on a single segment or globally in the complete vascular network. Global tortuosity quantification consists of automated segmentation and partition of retinal vessel network, local tortuosity measurement, and global tortuosity index derivation from weighted combination of local tortuosity values. This paper proposes several weighting schemes and evaluates their performance when combined with different local tortuosity indexes. We perform rank correlation analysis to find the global tortuosity quantification that is most consistent with the ophthalmologists. Our results show that local tortuosity indexes that are robust to variations in scale and number of sampling points provide the best performance. Furthermore, weighting scheme based on chord length yields better results than the one based on arc length. The combination of Tortuosity Density (TD) local index and Tortuosity Density Global (TDG) weighting scheme provides the highest consistency with ophthalmologists, with the average rank correlation coefficient of 0.98 (p-value $<0.03)$.
\end{abstract}

Keywords - retinopathy; local tortuosity; global tortuosity

\section{INTRODUCTION}

Changes in the morphology of the retinal blood vessel network commonly appear in diseases such as diabetic retinopathy [1], hypertensive retinopathy [2], retinopathy of prematurity [3], [4], and familial retinal arteriolar tortuosity abnormality [5]. In those diseases, the most common morphological change appearing in the retina is in the tortuosity of the vessel, making it appears increasingly more winded, turned, and twisted; due to either the weakening of the vessel wall or the abnormal growth of the vessel network [6]. Retinal vessel wall weakening can be attributed to prolonged hemodynamic disturbances such as fluctuations in blood sugar level and high blood pressure, leading to the development of the tortuous retinal vascular network. Retinal tortuosity changes often occur earlier than the common clinical manifestations of retinopathy, i.e., microaneurysm, hemorrhages, and exudates. Thus, increased tortuosity of retinal blood vessel segments may serve as the earliest indicator of hemodynamic-related retinopathy, such as the ones occurring from diabetes mellitus and hypertension [1], [2]. In retinopathy of prematurity and familial retinal arteriolar tortuosity abnormality, abnormal growth of vascular network appears respectively as increased tortuosity but diminishing vascular network coverage in specific areas of the retina [3] and increased tortuosity only in the arteriolar segments of the macular and peri-papillary area [4]. In both cases, regular observations of the retinal tortuosity are required to manage the diseases and determine the suitable cares.

Careful detection and measurement of the changes in the retinal vascular network tortuosity may contribute to preventing the undesired outcomes of retinopathy such as vision loss and blindness. For this purpose, tortuosity can be evaluated either locally in a vessel segment or globally in the entire blood vessel network. However, manual assessment of retinal vascular tortuosity is a time consuming and highly subjective task. An automated quantitative assessment may provide a more accurate and reproducible result, especially when time series observation on a specific area of the vessel network is required [1].

Some methods to quantify local tortuosity based on the calculation in digital retinal fundus imagery have been proposed in the literature [6]-14]. In general, all quantitative measurements of local tortuosity are performed on a smooth planar curve representing each single blood vessel segment [7]. The local tortuosity indexes are divided into four groups based on the primary determining feature incorporated by the algorithm, i.e., distance, curvature, angle variation and number of a twist. Each of these indexes has their characteristics when applied to vessels of different scales and orientation. In general, their performances are also influenced by the image resolution, since all calculations are performed on the series of coordinates representing a segment of the blood vessel. 
Clinical applications often demand global calculation of retinal vascular tortuosity, especially in the cases of hemodynamic-related retinopathy. In such necessity, the global tortuosity values are derived from the combination of the local tortuosity measurements in all vessel segments. Sharbaf, et al. defined global tortuosity as the mean of tortuosity values (MT) calculated on individual segments of the entire vessel network [8]. Hart, et al. used the weighted additivity (WA) method where they weight the local tortuosity of every vessel segment by its arc length to generate global tortuosity index [9]-[10]. In this case, arc length is defined as the actual length of the curve representing a vessel segment.

Global tortuosity index is intended to capture the overall quality of the blood vessel network. As such, an optimal weighting scheme that reasonably incorporates an individual contribution from each vessel segment, as well as the different complexity of the vascular network, is required. In this regard, the existing weighting schemes of MT and WA have their specific weaknesses. The MT weighting scheme cannot distinguish contributions from vessel segments of different length. For instance, a short vessel segment with high local tortuosity will dominate the value of global tortuosity measurement and mask the contributions from the remaining segments. The WA weighting scheme, on the other hand, fails to appreciate the different complexity of the vascular network, i.e., the different amount of branching points; because it only considers the crude arc length of each vessel segment. Under the WA weighting scheme, a vascular network with minimum and a maximum number of branching points but similar total crude arc lengths may have similar global tortuosity values.

The aim of this study is to find an optimal weighting scheme for quantitative global tortuosity measurement based on the existing local tortuosity indexes [6]-[14]. We propose three novel weighting schemes, i.e., combined mean tortuosity with weighted additivity (MTWA), weighted additivity on chord length (WAC) and tortuosity density global (TDG) to be used in global tortuosity calculation. We evaluate their agreement with qualitative assessment provided by an ophthalmologist and use the existing MT and WA weighting schemes as a benchmark. Our study offers a comparative quantitative analysis of new and existing schemes; all evaluated on the identical dataset.

This paper is structured as follows: After the introduction presented in section I, we describe the material and methods for global tortuosity quantification in section II and the results and discussions of our experiments in section III. The paper is concluded in section IV, where we also incorporate an additional insight into the potential future works related to this research.

\section{MATERIAL AND METHOD}

\section{A. Dataset}

Dataset used in this study is a compilation of digital retinal images provided by the Eye Image Analysis Research Group (EIARG), University of Mashhad, Iran [8]. Images were taken with a mydriatic digital fundus camera Topcon TRC.50EX with the field of view (FOV) of 50 degrees. The dataset contains ten retinal images from ten patients with the spatial resolution of $575 \times 479$ pixels as shown in Fig. 1. Five ophthalmologists provided manual global tortuosity ranking on the ten images obtained from the Retina Research Centre, Mashhad University of Medical Science, Khatam-alAnbya Hospital of Mashhad, Iran. The global tortuosity ranking was given as ordinal numbers from 1 to 10 . Each number was annotated to a corresponding retinal image, indicating the increasing order of qualitatively lowest to highest global tortuosity.

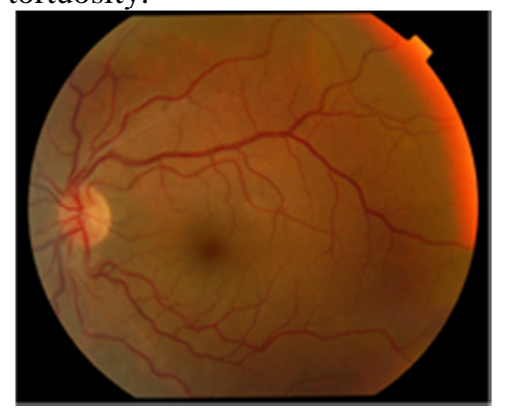

Fig. 1. Retinal image from EIARG dataset

\section{B. Global Tortuosity Evaluation}

Fig 2 illustrates the block diagram of global tortuosity evaluation in the retinal blood vessel. Before global tortuosity index calculation, the retinal image was processed to get the planar curve of all vessel segments in the entire blood vessel network. The identified network was then automatically partitioned into segments, in each of which a local tortuosity calculation was performed. The global tortuosity index was obtained by combining the local tortuosity calculation results from all segments according to specific index formulations. Finally, a Spearman's rank correlation coefficients $(\rho)$ are measured to analyze the consistency of the tortuosity ranking provided by our methods to the ground truth.

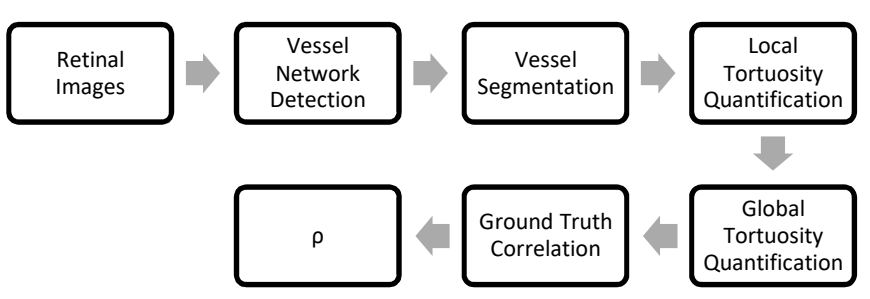

Fig. 2. The methodology of global tortuosity evaluation

1) Vessel Network Detection

The steps of vessel network detection are shown in Fig 3.

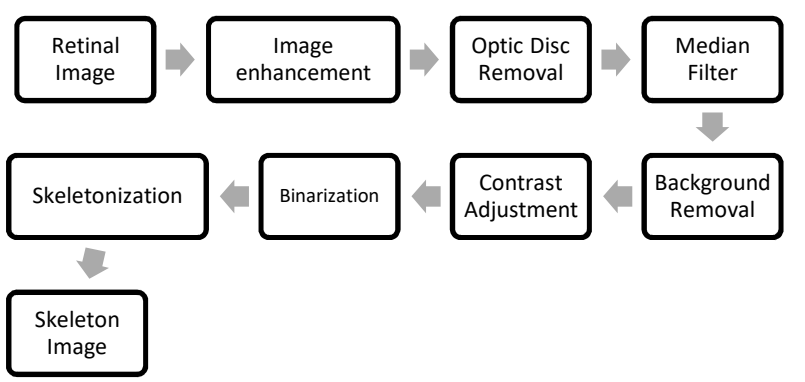

Fig. 3. The methodology of vessel network detection

The first step in the vessel network detection is retinal image enhancement. This step extracts the green channel of 
the retinal image represented by the RGB color channel system. Green channel is chosen because it provides the best vessels contrast. The enhanced green channel image is then complemented and processed to achieve a proportional distribution of pixel intensity. We use the standard implementation of histogram equalization to achieve intensity distribution proportionality. Fig 4 shows the result of this step.

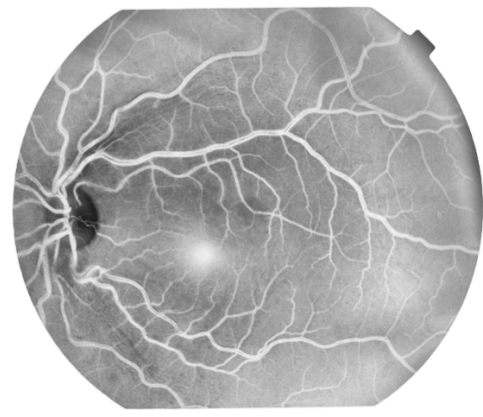

Fig. 4. Vessel network detection: the result of image enhancement

The next step is optic disc removal by subtracting the result of image enhancement with an image obtained from an opening morphological operation. We use a $3 \times 3$ structuring element in the shape of a ball followed by a median filter. To remove other objects except for the blood vessels in the entire image, we subtract this image with another result of an opening image operation; this time implemented using $15 \times 15$ structuring element in the shape of a disc. The result from background removal is shown in Fig 5 .

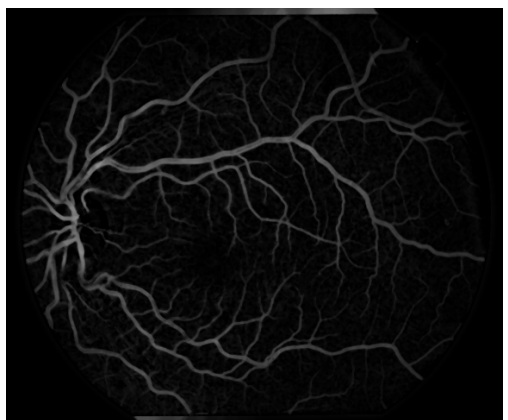

Fig. 5. Vessel network detection: the result of background removal

Contrast adjustment followed by binarization is further implemented to increase the visibility of blood vessels. In Fig 6, we show the vessel map obtained using the Otsu binarization algorithm.

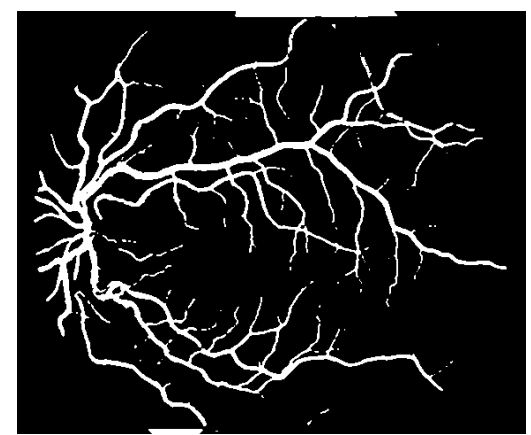

Fig. 6. Vessel network detection: initial binary vessel map
In order to find the centerline of each vessel in the vessel map, it is necessary to thin the vessel map and extract the vessel skeleton of one-pixel diameter as shown in Fig 7. This result is obtained by using morphological skeletonization method.

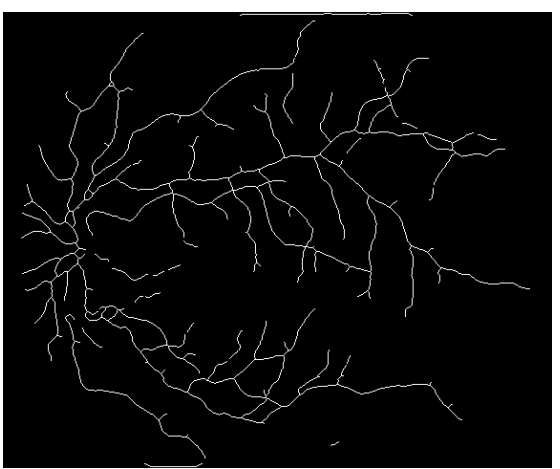

Fig. 7. Vessel network detection: skeletonized vessel map

\section{2) Vessel Segmentation}

In the next process, the vessel map is further partitioned into individual vessel segments to facilitate local tortuosity calculation. We define a vessel segment as a group of the pixel that extended either from an endpoint to the first bifurcation or crossover, between two bifurcations or crossovers, or between two endpoints. Vessel segmentation methodology is shown in Fig 8.

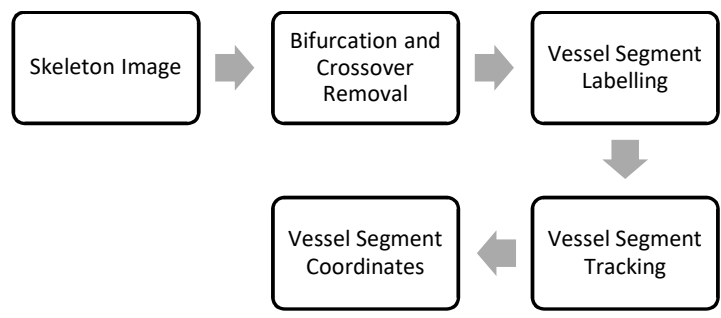

Fig. 8. The methodology of vessel partitioning into segments

The first step in vessel segmentation is to detect all bifurcation and crossover points from skeleton image. These points are identified within an 8-neighborhood pixel adjacency window. This window essentially compares a central pixel with its direct 8 neighboring pixels within a $3 \mathrm{x}$ 3 -pixel area. Such a window is positioned at every skeleton pixel, i.e., every pixel with an intensity value of " 1 " in the skeleton image.

Bifurcations points are defined as vessel pixels that have three 8-neighborhood adjacent pixels with a " 1 " intensity value. Crossover points are defined as vessel pixels that have four 8-neighborhood adjacent pixels with a "1" intensity value. As the bifurcation and crossover points are removed, the vessel network becomes divided into individual segments. Another morphological operation can thus determine the end and beginning of each segment. The corresponding morphological operation tries to identify endpoints, i.e., pixels that only have one 8-neighborhood adjacent pixel with a " 1 " intensity value. Fig 9 presents an example of bifurcations and crossover points identification, while Fig 10 presents the corresponding segmental endpoints. 


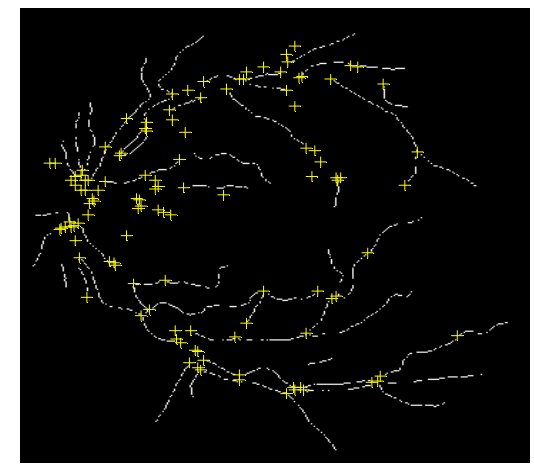

Fig. 9. Vessel Segmentation: skeleton image with bifurcation and crossover point (yellow crosses)

Each segments protruding from bifurcation and crossover points in the vessels network is then labeled to classify vessel segment object. Labeling is implemented with the connected component algorithm. It starts from an endpoint to another endpoint. Group of pixels that have the same label is defined as a vessel segment as shown in Fig 10. A number of the label indicates the number of a vessel segment in the vessel network. Tracking vessels pixel in each segment is necessary to ensure tortuosity measurement is started from an endpoint and finished at another endpoint. Pixel vessel segments are modeled as a continuous planar curve using cubic smoothing spline interpolation. Fig 11 is an example of two vessel segments produced by this process.

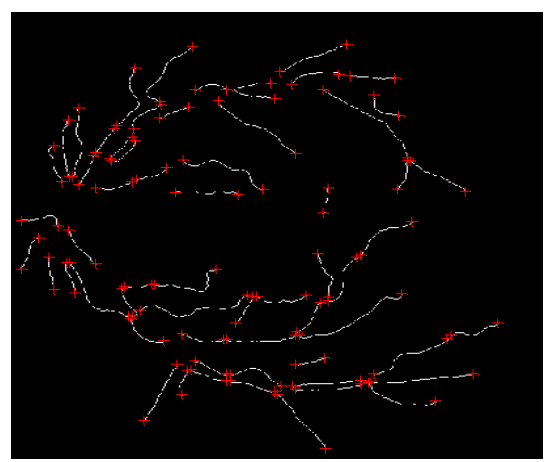

Fig. 10. Vessel segmentation: vessel labeling, where one vessel segment is defined as connected pixels between two red signs (end point)
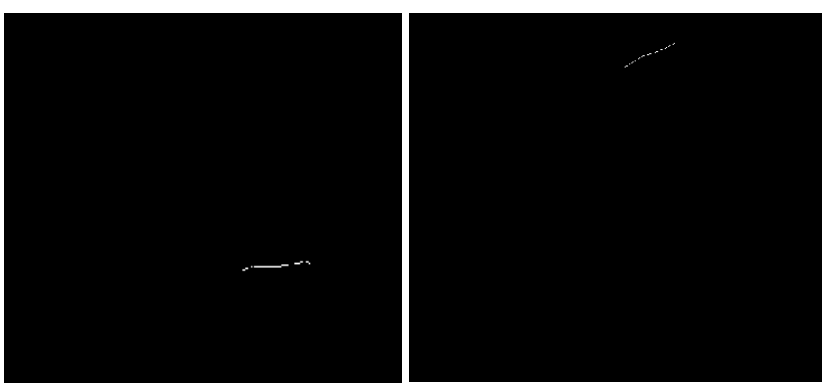

Fig. 11. Examples of individual vessel segments from the labeled image in Fig. 10

\section{3) Tortuosity Measurement}

a) Quantification of Local Tortuosity

Quantification of global tortuosity is based on the local tortuosity measures obtained in every vessels segment on a retinal vessel map. Vessel segment coordinates $S(x, y)$ serve as an input to the measurement of local tortuosity. We use twelve local tortuosity quantification methods, which can be divided in four groups, i.e. local, tortuosity based on distance, curvature, angle variation, and numbera of twists.

\section{- A method based on distance}

In distance-based methods, tortuosity index is derived from the ratio of the actual vessel length (arc length, $L_{a}$ ) and a straight line connecting the two end-points of a vessel segment (chord length, $L_{c}$ ). $L_{a}, L_{c}$, and tortuosity index $L_{a} / L_{c}$ are calculated using equations (1), (2) and (3), with $n$ is the number of points in a vessel segment [6]. Length calculations are intrinsically dependent on the number of sampling points used to represent the vessel segment coordinates. However, as two length measurements performed at similar scale are normalized against each other, such dependency is partially compensated.

$$
\begin{gathered}
L_{a}=\sum_{i=1}^{n-1} \sqrt{\left(x_{i+1}-x_{i}\right)^{2}+\left(y_{i+1}-y_{i}\right)^{2}} \\
L_{c}=\sqrt{\left(x_{n}-x_{i}\right)^{2}+\left(y_{n}-y_{i}\right)^{2}} \\
L a / L c=\frac{L_{a}(S)}{L_{c}(S)}-1
\end{gathered}
$$

- A method based on curvature

The curvature is a local curve behavior that informs the rate of directional change at every point $[x(t), y(t)]$ in a curve. It can be measured from derivatives of the coordinates as shown in (4).

$$
C(t)=\frac{y^{\prime \prime}(t) x^{\prime}(t)-x^{\prime \prime}(t) y^{\prime}(t)}{\left(\left(x^{\prime}(t)^{2}+y^{\prime}(t)^{2}\right)^{3 / 2}\right.}
$$

Curvature values measured along a vessel segment can be an indicator of tortuosity [10-11]. Integral of absolute curvature $(T C)$ in (5) and integral of squared curvature (TSC) in (6) are defined as local tortuosity indexes. Normalization with arc length $\left(L_{a}\right)$ or chord length $\left(L_{c}\right)$ allows comparison of various vessels with different lengths and scale variances. Patasius et al. [12] define local tortuosity index as the squared derivative of the curvature in every point over arc length, as in (7).

$$
\begin{aligned}
& T C(t)=\int_{t_{0}}^{t_{n}}|C(t)| d t \\
& \operatorname{TSC}(t)=\int_{t_{0}}^{t_{n}} C(t)^{2} d t \\
& D C I=\frac{1}{L_{a}} \int_{t_{0}}^{t_{n}}\left|\frac{d C(t)}{d t}\right|^{2} d t
\end{aligned}
$$

- A method based on angle variation

Mean Directional Angle Change $(M D A C)$ is the average of angle direction at every point in a vessel curve [13]. The 
local direction is obtained from the angle of a vector originated on a point in the curve $\left(p_{i}\right)$ and ended on the next and the previous $n$ points $\left(p_{i \pm n}\right)$ as define in (8). Local angle tortuosity index is measured using (9), where $N$ is the number of sample points in curve $S$. We perform the angle calculation on vectors formed at every 10 points, thus requiring a minimal segment length of 20 points. With the requirement of determining the range of angle calculation, the MDAC method becomes dependent on scale and the nuthe mber of sampling points used to represent the blood vessel segment.

$$
\begin{gathered}
v_{i+n}=p_{i+n}-p_{i}, v_{i-n}=p_{i-n}-p_{i} \\
M D A C=\frac{1}{N-2 . n} \sum_{i=1}^{n} \arccos \left(v_{i+n} \cdot v_{i-n}\right)
\end{gathered}
$$

Absolute Directional Angle Change (ADAC) considers local tortuosity of a vessel segment as the number of local direction angle $\left(\theta_{\mathrm{i}}\right)$ with the minimal value of $\pi / 6$ as in (10) [14]. Similar to MDAC, this method also requires the determination of range to calculate local direction angle, rendering the index to be influenced with scale and the number of sampling points as well.

$$
A D A C=\sum_{i=1}^{n}\left(\theta_{i} \geq \frac{\pi}{6}\right)
$$

\section{- A method based on the number of twists}

A number of twists is an important property to quantify tortuosity in a vessel segment. It can be calculated from the number of change in curvature sign or the number of zerocrossings from the second derivatives of curve coordinates. Bullitt et al. [15] proposed Inflection Count Metric (ICM) as a local tortuosity index based on numa ber of twists. It combines arc length and chord length ratio with numberthe of inflection point, $n_{i p}$ (twist) at a curve $S$ as in (11).

$$
I C M=\left(n_{i p}+1\right) \frac{L_{a}(S)}{L_{c}(S)}
$$

Tortuosity Density (TD) index evaluates vessel tortuosity in all sub-segments with constant curvature sign within a vessel segment [11]. Tortuosity index is defined as in (12) which $\mathrm{n}$ is numbthe er of sub-segment, $L_{a}(\mathrm{~S})$ is vessels arc length, $L_{a}\left(S_{i}\right)$ and $L_{c}\left(S_{i}\right)$ are sub-segment arc length and chord length respectively.

$$
T D=\frac{n-1}{n} \frac{1}{L_{a}(S)} \sum_{i=1}^{n}\left[\frac{L_{a}\left(S_{i}\right)}{L_{c}\left(S_{i}\right)}-1\right]
$$

Both methods incorporated the ratio of two length measurements, thus compensating their dependency on the scale and number of sampling points.

b) Quantification of Global Tortuosity: Weighting Schemes

Global tortuosity is obtained by combining any of local tortuosity indexes explained previously with a certain weighting scheme. The local tortuosity calculation is performed for every individual segment in the vascular network, and each resulted value is consequently weighted with a specific weighting scheme to produce a global tortuosity index.

In this study, we test two existing weighting schemes from the literature and propose three modified weighting schemes.

\section{- Mean Tortuosity (MT) [8]}

Global tortuosity is calculated as the mean of all local tortuosity in an image as in (13), where $m$ is the number of vessel segments, and $T_{L}$ is the local tortuosity index at each segment. This crude averaging method lacks the ability to weigh contributions from different lengths of blood vessel segments. Short segments with high local tortuosity are treated equally with longer segments with lower local tortuosity. The global tortuosity value may become dominated by the short segments with striking tortuosity, while the generic tortuosity of the rest of the vascular network become masked.

$$
M T=\frac{1}{m} \sum_{i=1}^{m} T_{L}
$$

\section{- Weighted Additivity (WA) [9],[10]}

The global tortuosity index by weighted additivity method weights the local tortuosity of each vessel segment by its arc length $\left(L_{a}\right)$ as in (14).

$$
W A=\frac{\sum_{i=1}^{m} L_{a}(i) \cdot T_{L}(i)}{\sum_{i=1}^{m} L_{a}(i)}
$$

This method cannot distinguish differences in the complexity of the vascular network structure, i.e., the differences in the number and density of branching points. Images with similar total arc length may have similar global tortuosity even when their vascular networks are structurally different.

\section{- Mean Tortuosity with Weighted Additivity (MTWA)}

Based on the weaknesses of the MT and WA weighting schemes, we propose to combine the mean tortuosity and weighted additivity algorithm as in (15). By considering both the number of segments and the length of segments, we hope to obtain a more accurate weighting scheme.

$$
M T W A=\frac{\sum_{i=1}^{m} L_{a}(i) \cdot T_{L}(i)}{m \cdot \sum_{i=1}^{m} L_{a}(i)}
$$

\section{- Weighted Additivity on Chord (WAC)}

We also attempt to modify the weighted additivity algorithm from originally formulated on arc length $\left(L_{a}\right)$ to chord length $\left(L_{c}\right)$ as given in (16). It is known that both length measurements are sensitive to scale and number of sampling points. However, the extent of such influence can be expected to be more proportional in chord than in arc length calculation. In chord length calculation (i.e., Euclidean distance), all segments will be compromised 
equally; while in arc length calculation (actual vessel length), the segments with higher tortuosity will be affected much more than the ones with lower tortuosity.

$$
W A C=\frac{\sum_{i=1}^{m} L_{c}(i) \cdot T_{L}(i)}{\sum_{i=1}^{m} L_{c}(i)}
$$

\section{- Tortuosity Density Global (TDG) index}

This method extends the local tortuosity density (TD) algorithm in (12) to be used in the global calculation. TD algorithm considers a local segment as a sequential combination of shorter sub-segments. Our proposed TDG index treats individual local vessel segments as TD subsegments. In (17), $m$ is the number of vessel segment, arc over chord length ratio is used as local tortuosity indexes, and the arc length of the entire vessel network is used as a normalization factor.

$$
T D G=\frac{\sum_{i=1}^{m} T_{L}(i)}{m \cdot \sum_{i=1}^{m} L_{a}(i)}
$$

\section{RESULT AND DISCUSSION}

We evaluate the performance of our global tortuosity indexes by measuring the Spearman's rank correlation coefficient $(\rho)$ between the image tortuosity rankings provided by our quantitative methods and the qualitative rankings provided by five independent ophthalmologists. We first assess the correspondence between each ophthalmologist to find out whether they are in good agreements. Table I shows high positive correlation $(0.76 \leq$ $\rho \leq 0.96$, correlation range $R_{C}=0.2$ ) between the five ophthalmologists $\left(D_{1}, D_{2}, D_{3}, D_{4}\right.$, and $\left.D_{5}\right)$ with significance level at 0.002 . These values inform that the ophthalmologist assessments are in good agreement with each other; therefore their rankings can be used as a reference to determine the performance of our proposed method. The range of correlation values can be considered as the representative range of inter-observer variations commonly found in subjective assessments.

TABLE I

SPEARMAN'S RANK CORRELATION COEFFICIENT BETWEEN FIVE OPHTHALMOLOGISTS $\left(\mathrm{D}_{1}-\mathrm{D}_{5}\right)$

\begin{tabular}{|c|c|}
\hline Ophthalmologists & $\rho(p$-value <0.02) \\
\hline$D_{12}$ & 0.77 \\
\hline$D_{13}$ & 0.94 \\
\hline$D_{14}$ & 0.93 \\
\hline$D_{15}$ & 0.86 \\
\hline$D_{23}$ & 0.76 \\
\hline$D_{24}$ & 0.88 \\
\hline$D_{25}$ & 0.88 \\
\hline$D_{34}$ & 0.96 \\
\hline$D_{35}$ & 0.86 \\
\hline$D_{45}$ & 0.93 \\
\hline
\end{tabular}

With the combinations of twelve local tortuosity indexes, five weighting schemes, and five individual rankings by ophthalmologists, we ended up with 60 pairs of Spearman's rank correlation analyses per weighting scheme, or 300 pairs of global tortuosity correlation analyses in total. We present the results of each 60 pairs of correlation analysis in the following Table II - Table VI, where each table corresponds to a specific weighting scheme.

We decided to perform our correlation analysis on each ophthalmologist assessment instead of combining the five manual rankings into one. In this way, we can observe how our quantitative measurements perform with varying qualitative assessments provided by an ophthalmologist. The range of the lowest and highest inter-ophthalmologist correlations from Table 1 can further be compared to the range of the lowest and highest correlations for each tested quantitative index in Table II - Table VI to investigate whether quantitative methods are more consistent than qualitative evaluations.

TABLE II

SPEARMAN'S RANK CORRELATION COEFFICIENT FROM MEAN TORTUOSITY

\begin{tabular}{|c|c|c|c|c|c|c|c|}
\hline \multirow{2}{*}{\multicolumn{2}{|c|}{ Method }} & \multicolumn{6}{|c|}{ Correlation $\rho(p$-value $<0.03)$} \\
\hline & & \multirow{2}{*}{$\begin{array}{c}\boldsymbol{D}_{\mathbf{1}} \\
0.88\end{array}$} & \multirow{2}{*}{$\begin{array}{c}\boldsymbol{D}_{\mathbf{2}} \\
0.74\end{array}$} & \multirow{2}{*}{$\begin{array}{c}\boldsymbol{D}_{3} \\
0.85\end{array}$} & \multirow{2}{*}{$\begin{array}{c}\boldsymbol{D}_{\mathbf{4}} \\
0.82\end{array}$} & \multirow{2}{*}{$\begin{array}{c}\boldsymbol{D}_{\mathbf{5}} \\
0.76\end{array}$} & \multirow{2}{*}{$\begin{array}{c}\text { Mean } \\
0.81\end{array}$} \\
\hline Distance & $L_{a} / L_{c}$ & & & & & & \\
\hline \multirow{7}{*}{ Curvature } & $T C$ & 0.53 & 0.29 & 0.43 & 0.33 & 0.25 & 0.37 \\
\hline & $T S C$ & 0.81 & 0.62 & 0.78 & 0.72 & 0.65 & 0.72 \\
\hline & $T C / L_{a}$ & 0.75 & 0.54 & 0.67 & 0.59 & 0.54 & 0.62 \\
\hline & $T S C / L_{a}$ & 0.93 & 0.76 & 0.86 & 0.81 & 0.83 & 0.84 \\
\hline & $T C / L_{c}$ & 0.73 & 0.55 & 0.69 & 0.6 & 0.62 & 0.64 \\
\hline & $T S C / L_{c}$ & 0.94 & 0.78 & 0.92 & 0.86 & 0.79 & 0.86 \\
\hline & $D C I$ & 0.85 & 0.55 & 0.83 & 0.75 & 0.65 & 0.73 \\
\hline \multirow{2}{*}{$\begin{array}{c}\text { Angle } \\
\text { variation }\end{array}$} & $M D A C$ & 0.35 & 0.21 & 0.32 & 0.25 & 0.21 & 0.27 \\
\hline & $A D A C$ & 0.02 & 0.17 & 0.01 & 0.11 & 0.23 & 0.11 \\
\hline \multirow{2}{*}{$\begin{array}{l}\text { Number } \\
\text { of twist }\end{array}$} & ICM & 0.35 & 0.18 & 0.33 & 0.21 & 0.09 & 0.23 \\
\hline & $T D$ & 0.94 & 0.81 & 0.89 & 0.91 & 0.87 & 0.88 \\
\hline
\end{tabular}
METHOD AND GROUND TRUTH

Table II illustrates the Spearman's rank correlation coefficient of tortuosity ranking from Mean Tortuosity (MT) weighting scheme compared to ground truth. Our experiment shows that $T D$ is the best local tortuosity method to be used in conjunction with the MT weighting scheme ( $\rho_{\text {mean }}=0.88$, $0.81 \leq \rho \leq 0.94, R_{C}=0.13$ ). This result is in conjunction with the characteristics of $T D$ inda the ex, which is more robust to variances in scale and vessel sampling points.

TABLE III

SPEARMAN'S RANK CORRELATION COEFFICIENT FROM WEIGHTED

\begin{tabular}{|c|c|c|c|c|c|c|c|}
\hline \multirow{2}{*}{\multicolumn{2}{|c|}{ Method }} & \multicolumn{6}{|c|}{ Correlation $\rho(p$-value $<0.03)$} \\
\hline & & \multirow{2}{*}{$\begin{array}{c}\boldsymbol{D}_{\mathbf{1}} \\
0.71\end{array}$} & \multirow{2}{*}{$\begin{array}{c}\boldsymbol{D}_{\mathbf{2}} \\
0.84\end{array}$} & \multirow{2}{*}{$\begin{array}{c}\boldsymbol{D}_{3} \\
0.77\end{array}$} & \multirow{2}{*}{$\frac{\boldsymbol{D}_{\mathbf{4}}}{0.84}$} & \multirow{2}{*}{$\begin{array}{c}\boldsymbol{D}_{\mathbf{5}} \\
0.77\end{array}$} & \multirow{2}{*}{$\begin{array}{c}\text { Mean } \\
0.79\end{array}$} \\
\hline Distance & $L_{a} / L_{c}$ & & & & & & \\
\hline \multirow{7}{*}{ Curvature } & $T C$ & 0.61 & 0.4 & 0.77 & 0.66 & 0.45 & 0.58 \\
\hline & $T S C$ & 0.81 & 0.78 & 0.93 & 0.9 & 0.77 & 0.84 \\
\hline & $T C / L_{a}$ & 0.95 & 0.93 & 0.96 & 0.98 & 0.96 & 0.96 \\
\hline & $T S C / L_{a}$ & 0.91 & 0.89 & 0.93 & 0.96 & 0.94 & 0.93 \\
\hline & $T C / L_{c}$ & 0.91 & 0.89 & 0.93 & 0.96 & 0.94 & 0.93 \\
\hline & $T S C / L_{c}$ & 0.91 & 0.89 & 0.93 & 0.96 & 0.94 & 0.93 \\
\hline & $D C I$ & 0.95 & 0.93 & 0.95 & 0.97 & 0.93 & 0.95 \\
\hline \multirow{2}{*}{$\begin{array}{c}\text { Angle } \\
\text { variation }\end{array}$} & $M D A C$ & 0.11 & 0.39 & 0.16 & 0.33 & 0.45 & 0.29 \\
\hline & $A D A C$ & 0.01 & -0.3 & -0.02 & -0.18 & -0.28 & -0.15 \\
\hline \multirow{2}{*}{$\begin{array}{l}\text { Number } \\
\text { of twist }\end{array}$} & $I C M$ & 0.54 & 0.19 & 0.41 & 0.27 & 0.12 & 0.31 \\
\hline & $T D$ & 0.86 & 0.85 & 0.94 & 0.96 & 0.82 & 0.89 \\
\hline
\end{tabular}
ADDITIVITY METHOD AND GROUND TRUTH 
Table III shows the Spearman's rank correlation coefficient of tortuosity ranking from Weighted Additivity (WA) weighting scheme as opposed to ground truth. In our experiment, $T C / L_{a}$ consistently shows the highest correlation with all five ophthalmologists. The $T C / L_{a}$ is thus the best local tortuosity index to be used in conjunction with WA, with $\rho_{\text {mean }}=0.96\left(0.93 \leq \rho \leq 0.98, R_{C}=0.05\right)$. This result is better than the previous weighting scheme, since it takes into account the length of the vessel segments involved.

TABLE IV

SPEARMAN'S RANK CORRELATION COEFFICIENT FROM MEAN TORTUOSITY WEIGHTED ADDITIVITY METHOD AND GROUND TRUTH

\begin{tabular}{|c|c|c|c|c|c|c|c|}
\hline \multirow{2}{*}{ Method } & \multicolumn{7}{|c|}{ Correlation $\boldsymbol{\rho}$ (p-value $<\mathbf{0 . 0 3 )}$} \\
\cline { 2 - 8 } & $\boldsymbol{D}_{\mathbf{1}}$ & $\boldsymbol{D}_{\mathbf{2}}$ & $\boldsymbol{D}_{\mathbf{3}}$ & $\boldsymbol{D}_{\mathbf{4}}$ & $\boldsymbol{D}_{\mathbf{5}}$ & Mean \\
\hline Distance & $L_{a} / L_{c}$ & 0.77 & $\mathbf{0 . 8 2}$ & 0.84 & 0.87 & 0.79 & 0.82 \\
\hline \multirow{4}{*}{ Curvature } & $T C$ & 0.22 & -0.1 & 0.23 & 0.05 & -0.07 & 0.07 \\
\cline { 2 - 8 } & $T S C$ & 0.77 & 0.54 & 0.78 & 0.68 & 0.56 & 0.67 \\
\cline { 2 - 8 } & $T C / L_{a}$ & 0.71 & 0.49 & 0.71 & 0.61 & 0.56 & 0.62 \\
\cline { 2 - 8 } & $T S C / L_{a}$ & 0.81 & 0.66 & 0.87 & 0.79 & 0.73 & 0.77 \\
\cline { 2 - 8 } & $T C / L_{c}$ & 0.7 & 0.52 & 0.71 & 0.65 & 0.5 & 0.62 \\
\cline { 2 - 8 } & $T S C / L_{c}$ & 0.86 & 0.69 & 0.93 & 0.85 & 0.81 & 0.83 \\
\cline { 2 - 8 } & $D C I$ & 0.78 & 0.52 & 0.82 & 0.7 & 0.62 & 0.69 \\
\hline \multirow{4}{*}{$\begin{array}{c}\text { Angle } \\
\text { variation }\end{array}$} & $M D A C$ & -0.12 & -0.35 & -0.14 & -0.29 & -0.38 & -0.26 \\
\cline { 2 - 8 } $\begin{array}{c}\text { Number } \\
\text { of twist }\end{array}$ & $A D A C$ & -0.12 & -0.35 & -0.14 & -0.29 & -0.38 & -0.26 \\
\cline { 2 - 8 } & $I C M$ & 0.07 & -0.23 & 0.04 & -0.12 & -0.23 & -0.09 \\
\hline
\end{tabular}

Table IV describes the Spearman's rank correlation coefficient of tortuosity ranking from the Mean Tortuosity Weighted Additivity (MTWA) weighting scheme compared to ground truth. MTWA method gives the best performance when used together with $T D$ local tortuosity index, giving $\rho_{\text {mean }}=0.84\left(0.77 \leq \rho \leq 0.92, R_{C}=0.15\right)$. The tortuosity density index itself has contained normalization of arc length to chord length. Therefore weighting with arc length compensates for the sampling point dependency of the arc length and weighting with segment number further completes the weighting scheme.

TABLE V

SPEARMAN'S RANK CORRELATION COEFFICIENT FROM WEIGHTED ADDITIVITY CHORD METHOD AND GROUND TRUTH

\begin{tabular}{|c|c|c|c|c|c|c|c|}
\hline \multirow{2}{*}{ Method } & \multicolumn{7}{|c|}{ Correlation $\boldsymbol{\rho}$ (p-value $<\mathbf{0 . 0 3})$} \\
\cline { 2 - 8 } & $\boldsymbol{D}_{\mathbf{1}}$ & $\boldsymbol{D}_{\mathbf{2}}$ & $\boldsymbol{D}_{\mathbf{3}}$ & $\boldsymbol{D}_{\mathbf{4}}$ & $\boldsymbol{D}_{\mathbf{5}}$ & Mean \\
\hline Distance & $L_{a} / L_{c}$ & 0.78 & 0.82 & 0.84 & 0.89 & 0.85 & 0.84 \\
\hline \multirow{4}{*}{\begin{tabular}{c} 
Curvature \\
\cline { 2 - 8 }
\end{tabular}} & $T C$ & 0.62 & 0.39 & 0.75 & 0.63 & 0.43 & 0.56 \\
\cline { 2 - 8 } & $T S C$ & 0.8 & 0.61 & 0.91 & 0.84 & 0.76 & 0.78 \\
\cline { 2 - 8 } & $T C / L_{a}$ & 0.8 & 0.61 & 0.91 & 0.84 & 0.76 & 0.78 \\
\cline { 2 - 8 } & $T S C / L_{a}$ & 0.95 & 0.92 & 0.94 & 0.96 & 0.92 & 0.94 \\
\cline { 2 - 8 } & $T C / L_{c}$ & 0.86 & 0.84 & 0.93 & 0.94 & 0.87 & 0.89 \\
\cline { 2 - 8 } & $T S C / L_{c}$ & 0.98 & 0.86 & 0.93 & 0.96 & 0.89 & 0.92 \\
\cline { 2 - 8 } & $D C I$ & 0.98 & 0.96 & 0.96 & 0.98 & 0.95 & $\mathbf{0 . 9 7}$ \\
\hline \multirow{3}{*}{$\begin{array}{c}\text { Angle } \\
\text { variation }\end{array}$} & $M D A C$ & 0.08 & 0.37 & 0.21 & 0.35 & 0.46 & 0.29 \\
\cline { 2 - 8 } of twist & $A D A C$ & 0.04 & -0.28 & 0.04 & -0.12 & -0.28 & -0.12 \\
\cline { 2 - 8 } & $I C M$ & -0.52 & -0.75 & -0.57 & -0.71 & -0.75 & -0.66 \\
\cline { 2 - 8 } & $T D$ & 0.84 & 0.78 & 0.92 & 0.94 & 0.81 & 0.86 \\
\hline
\end{tabular}

Table V gives the Spearman's rank correlation coefficient of tortuosity ranking from the Weighted Additivity Chord (WAC) weighting scheme as opposed to the ground truth. Our experiments show that DCI local tortuosity index gives the highest correlations with $\mathrm{D}_{1}, \mathrm{D}_{2}, \mathrm{D}_{4}$ and $\mathrm{D}_{5}$. The $D C I$ is shown to be the best local tortuosity method to be used in conjunction with the WAC algorithm, with $\rho_{\text {mean }}=0.97$ $\left(0.95 \leq \rho \leq 0.98, R_{C}=0.03\right)$. The DCI local tortuosity index are normalized to arc length, therefore adding weighting sca heme based on chord length completes the global tortuosity index.

Table VI illustrates the Spearman's rank correlation coefficient of tortuosity ranking from the Tortuosity Density Global (TDG) weighting scheme as compared to ground truth. TDG weighting scheme gives the best performance when used in conjunction with TD local tortuosity index, with $\rho_{\text {mean }}=0.98\left(0.96 \leq \rho \leq 0.99, R_{C}=0.05\right)$. The result can be expected since there is a natural match between the TD and the TDG weighting scheme, with the latter being the direct extension of the TD algorithm.

TABLE VI

SPEARMAN'S RANK CORRELATION COEFFICIENT FROM THE TORTUOSITY DENSITY GLOBAL METHOD AND GROUND TRUTH

\begin{tabular}{|c|c|c|c|c|c|c|c|}
\hline \multirow{2}{*}{\multicolumn{2}{|c|}{ Method }} & \multicolumn{6}{|c|}{ Correlation $\rho(p$-value $<0.03)$} \\
\hline & & \multirow{2}{*}{$\begin{array}{c}\boldsymbol{D}_{\mathbf{1}} \\
0.89\end{array}$} & \multirow{2}{*}{$\begin{array}{c}\boldsymbol{D}_{2} \\
0.97\end{array}$} & \multirow{2}{*}{$\begin{array}{c}\boldsymbol{D}_{3} \\
0.94\end{array}$} & \multirow{2}{*}{$\begin{array}{c}\boldsymbol{D}_{\mathbf{4}} \\
0.95\end{array}$} & \multirow{2}{*}{$\begin{array}{c}\boldsymbol{D}_{\mathbf{5}} \\
0.85\end{array}$} & \multirow{2}{*}{$\frac{\text { Mean }}{0.92}$} \\
\hline Distance & $L_{a} / L_{c}$ & & & & & & \\
\hline \multirow{7}{*}{ Curvature } & $T C$ & 0.71 & 0.56 & 0.71 & 0.61 & 0.51 & 0.62 \\
\hline & $T S C$ & 0.82 & 0.69 & 0.88 & 0.79 & 0.66 & 0.77 \\
\hline & $T C / L_{a}$ & 0.62 & 0.42 & 0.54 & 0.44 & 0.43 & 0.49 \\
\hline & $T S C / L_{a}$ & 0.95 & 0.87 & 0.99 & 0.94 & 0.88 & 0.93 \\
\hline & $T C / L_{c}$ & 0.93 & 0.79 & 0.87 & 0.81 & 0.78 & 0.84 \\
\hline & $T S C / L_{c}$ & 0.95 & 0.87 & 0.99 & 0.94 & 0.88 & 0.93 \\
\hline & $D C I$ & 0.93 & 0.85 & 0.96 & 0.89 & 0.78 & 0.88 \\
\hline \multirow{2}{*}{$\begin{array}{c}\text { Angle } \\
\text { variation }\end{array}$} & $M D A C$ & 0.24 & -0.09 & 0.22 & 0.04 & -0.06 & 0.07 \\
\hline & $A D A C$ & -0.09 & -0.36 & -0.16 & -0.3 & -0.38 & -0.26 \\
\hline \multirow{2}{*}{$\begin{array}{l}\text { Number } \\
\text { of twist }\end{array}$} & $I C M$ & -0.14 & -0.33 & -0.31 & -0.32 & -0.37 & -0.29 \\
\hline & $T D$ & 0.99 & 0.96 & 0.99 & 0.97 & 0.97 & 0.98 \\
\hline
\end{tabular}

In global tortuosity quantification, vessel segments in a vessel map will have different scales. However, our local tortuosity calculations implement a single fixed scale in their calculations. The performance of local tortuosity methods such as TC, TSC, MDAC, ADAC, and ICM are naturally very dependent on scales and number of sampling points. This characteristic explains the lower correlation coefficient values obtained with these methods compared to the rests.

Global tortuosity indexes derived from local indexes which contain normalized ratio to either arc length, chord length or arc length over chord length tends to fare much better than the rest due to its increased robustness to the variation of scales and number of sampling points. Within these categories are the distance-based, normalized curvaturebased, and tortuosity density local indexes (i.e., $L_{a} / L_{c}, T C / L_{a}$, $\left.T C / L_{c}, T S C / L_{a}, T S C / L_{c}, T D\right)$.

The best global tortuosity indexes for each of the five variations of weighting schemes generally provide a smaller range of correlation values compared to the range of interophthalmologist agreements (correlation range $R_{C}=0.2$ ). The correlation ranges for global tortuosity indexes lie within the value of $R_{C} \leq 0.15$ for $M T$ and $M T W A$ and $R_{C} \leq 0.05$ for $W A$, $W A C$, and TD. This result means that the other four weighting schemes have successfully increased consistency on the evaluation of global tortuosity indexes.

Among the novel three weighting schemes that we introduced, i.e., the MTWA, WAC, and $T D G$, the most optimum result is given by the combination of tortuosity 
density $(T D)$ local index and tortuosity density global (TDG) weighting scheme, with an average Spearman's rank correlation coefficient of 0.98 . The DCI local index combined with the WAC weighting scheme came second with an average Spearman's rank correlation coefficient of 0.97 , while the TD local index and MTWA weighting scheme combination yield an average Spearman's rank correlation coefficient of 0.84 . The $T D G$ weighting scheme incorporates both the arc over chord length and the segment number normalization, thus presenting the weighting scheme with the complete inclusion of global traits of the retinal vascular network.

Our result is also affected by errors introduced in the segmentation and partitioning of blood vessel network. Common problems in digital retinal images such as noise due to non-homogeneous illumination remain a challenging problem in automated vessel segmentation. Vessel partitioning is hindered by the missing information of depth in the conventional two-dimensional fundus images, thus making segmentation of arteries and venous segments troublesome. Unfortunately, our dataset was not equipped with manually traced skeletons of the retinal vascular network as well as manually annotated identification of blood vessel segments. We, therefore, are unable to evaluate the performance of our blood vessel segmentation and partitioning algorithms.

Last, we only evaluated our algorithms on a minimal number of images. The result of methods described in this paper may be different as more images are included and if the images were of different resolutions. The main weakness of our quantitative tortuosity calculations is that it examines all scales and calibers of vessels with a similar fixed number of sampling points, thus providing different measurement fidelity for smaller and larger vessel segments. It is, however, an inherent problem of all quantitative tortuosity measurement indexes available to date.

\section{CONCLUSIONS}

From our experimental results, we conclude that local tortuosity indexes which values are sensitive to scale and number of sampling points (i.e. TC, TSC, MDAC, ADAC) are less appropriate for global tortuosity quantification than those who are more robust to such variations (i.e. $\mathrm{La} / \mathrm{Lc}$, TC/La, TC/Lc, TSC/La, TSC/Lc, TD). This nature stemmed from the inherently unaccounted variations of scale in the calculation of local tortuosity indexes. We also conclude that for retinal vasculature, global weighting scheme based on chord length is better than those that are based on arc length, because the influence of scales and sampling points in chord length is more proportional than in the arc length. The best result, however, is produced when length normalization is combined with segment number normalization as implemented in the TDG weighting scheme. The combination of TD as local tortuosity measure and TDG weighting scheme provides the highest average Spearman's rank correlation coefficient of 0.98 with five ophthalmologists.

Future works of this study are to improve the segmentation and partition of blood vessels network as well as to evaluate the algorithms on a more substantial number of images. It is also crucial to improve the local tortuosity index methodology to proportionally take into account the variations in scale and number of sampling points. With a more robust and accurate local tortuosity measurement, it is more likely to achieve an equally robust and accurate global tortuosity index.

\section{REFERENCES}

[1] M. B. Sasongko, T.Y. Wong, T.T. Nguyen, C.Y. Cheung, J.E. Shaw, R. Kawasaki, E.L. Lamoureux, and J.J. Wang. "Retinal vessel tortuosity and its relation to traditional and novel vascular risk markers in persons with diabetes." Curr Eye Res, vol.41(4), pp.551557, 2016.

[2] M. Cavallari, C. Stamile, R. Umeton, F. Calimeri, and F. Orzi. "Novel method for automated analysis of retinal images: results in subjects with hypertensive retinopathy and CADASIL." Biom Res Intl, 2015.

[3] F. Oloumi, R. M. Rangayyan, and A. L. Ells. "Computer-aided diagnosis of retinopathy in retinal fundus images of preterm infants via quantification of vascular tortuosity." J of Med Img, vol.3(4), pp. 044.505, 2016.

[4] F. Oloumi, R. M. Rangayyan, P. Casti, and A. L. Ells. "Computeraided diagnosis of plus disease via measurement of vessel thickness in retinal fundus images of preterm infants." Comp in Biol and Med, vol.66, pp.316-329, 2015.

[5] J.C. Zenteno, J. Crespí, B. Buentello-Volante, J.A. Buil, F. Bassaganyas, J.L. Vela-Segarra, and M.T. Marieges. "Next-generation sequencing uncovers a missense mutation in COL4A1 as the cause of familial retinal arteriolar tortuosity". Graefe's Arch. Clin. Exp. Ophthalmol, vol.252(11), pp. 1789-1794, 2015.

[6] W. Lotmar, A. Freiburghaus, and D. Bracher, "Measurement of vessel tortuosity on fundus photographs," Graefe's Arch. Clin. Exp. Ophthalmol, vol. 211, pp. 49-57, 1979.

[7] T. Mapayi, J. R. Tapamo, S. Viriri, and A. O. Adio, "Automatic retinal vessel detection and tortuosity measurement," Image Anal Stereol, 35:117-135, 2016.

[8] M. A. Sharbaf, H. R. Pourreza, T. Banaee. "A novel curvature based algorithm for automatic grading of retinal blood vessel tortuosity," IEEE J of Biomed and Health Inform. 2015.

[9] W. E. Hart, M. Goldbaum, P. Kube, and M.R. Nelson, "Automated measurement of retinal vascular tortuosity," AMIA Annual Fall Symposium Proceedings, 1997.

[10] W. E. Hart, M. Goldbaum, B. Cote, and P. Kube, "Measurement and classification of retinal vascular tortuosity," Int. J. Med. Informatics, vol.53, no. 23, pp. 239-52, Feb. 1999.

[11] E. Grisan, M. Foracchia, and A. Ruggeri, "A novel method for the automatic grading of retinal vessel tortuosity," IEEE Trans. on Med Img, vol.27, no.3, pp.310-319, 2008.

[12] M. Patasius, V. Marozas, D. Jegelevicius, and A. Lukosevicius, "Evaluation of tortuosity of eye blood vessels using the integral of the square of derivative of curvature," IFMBE Proc. 3rd Eur. Med. Biol. Eng. Conf. (EMBEC05), vol. 11. 2005.

[13] K. V. Chandrinos, M. Pilu, R. B. Fisher, and P. Trahanias, "Image processing techniques for the quantification of atherosclerotic changes," DAI Research paper, 1998.

[14] K. G. Goh, H. Wynne, M. L. Lee, and H. Wang, K. Cios, Ed., "Adris: an automatic diabetic retinal image screening system," Med. Data Min. Knowledge Discovery, pp. 181-21, 2001.

[15] E. Bullitt, G. Gerig, S. M. Pizer, W. Lin, and S.R Aylward, "Measuring tortuosity of the intracerebral vasculature from MRA image," Med. Image Anal., vol. 9, pp. 1163-1171, Sep. 2003. 